\title{
Expression of Jun and Fos proteins in ovarian tumors of different malignant potential and in ovarian cancer cell lines
}

\author{
SIBYLL HEIN $^{1 *}$, SVEN MAHNER $^{1 *}$, CHRISTINE KANOWSKI $^{1}$, \\ THOMAS LÖNING ${ }^{2}$, FRITZ JÄNICKE ${ }^{1}$ and KARIN MILDE-LANGOSCH ${ }^{1}$
}

${ }^{1}$ Department of Gynecology and Gynecologic Oncology, University Medical Center Hamburg-Eppendorf, Martinistrasse 52, 20246 Hamburg; ${ }^{2}$ Institute of Pathology, Albertinen Hospital Hamburg, Fangdieckstrasse 75a, 22547 Hamburg, Germany

Received February 11, 2009; Accepted March 26, 2009

DOI: $10.3892 /$ or_00000422

\begin{abstract}
Jun and Fos proteins form the transcription factor activating protein 1 (AP-1). They play a role in cell proliferation, malignant transformation and invasion in various tumors. The aim of the current study was to characterize the role of AP-1 in ovarian cancer. Fifty-six ovarian tumors of different invasive potential including 13 metastases as well as 5 ovarian cancer cell lines were analyzed by Western blot analysis regarding their expression of pc-Jun, Jun B, Jun D, c-Fos, Fos B, Fra-1 and Fra-2. In addition, invasion, cell proliferation and migration assays were performed. The expression of pc-Jun, Jun B, Jun D and Fra-2 was higher in invasive cancer compared to benign tumors. In metastases, c-Fos and Fos B expression was significantly lower than in the respective primary ovarian carcinomas. The invasive and proliferative potential of the cells was not associated with AP-1 protein expression. These results suggest that AP-1 proteins are differentially expressed in benign ovarian tumors, tumors with low malignant potential and epithelial ovarian carcinomas and metastases. No correlation with the proliferative and invasive potential of ovarian cancer cell lines could be found.
\end{abstract}

\section{Introduction}

Epithelial ovarian cancer derives from malignant transformation of the ovarian surface epithelium, which is similar to the peritoneal mesothelium. It accounts for the highest tumor-related mortality rate among all women with gynecologic malignancies. The American Cancer Society estimates 23,000 new cases of ovarian cancer each year in

Correspondence to: Dr Karin Milde-Langosch, Department of Gynecology and Gynecologic Oncology, University Medical Center Hamburg-Eppendorf, Martinistrasse 52, 20246 Hamburg, Germany E-mail: milde@uke.uni-hamburg.de

*Contributed equally

Key words: ovarian cancer, activating protein 1, Jun, Fos, expression, borderline tumor, metastasis the United States (1). Most patients present with advanced disease at initial diagnosis. Despite aggressive surgical cytoreduction and platinum-based chemotherapy, $70 \%$ of the patients will relapse and finally die of their disease (2).

Malignant transformation and progression are characterized by a variety of cellular functions including deregulated cell proliferation, suppression of apoptosis, increased invasive potential, generation of angiogenic signals and formation of metastases. All these processes involve changes in transcriptional regulation, implying that insight into this complex regulation may help to develop more targeted therapeutic strategies (3). Cell invasion depends on the coordinate expression and function of a variety of gene products conferring the ability of tumor cells to break away from the primary tumor and invade through the basal membrane and extracellular matrix (4). The transcription factor complex 'activating protein 1' (AP-1) plays a central role in gene regulation during these processes $(5,6)$.

AP-1 is composed of a variety of protein dimers from the Fos, Jun and ATF family. While Fos proteins (c-Fos, Fos B, Fra-1, Fra-2) can only form heterodimers with members of the Jun family, the Jun proteins (pc-Jun, Jun B, Jun D) can form both homodimers and heterodimers with Fos and ATF family proteins. These dimers generate transcriptionally active complexes that locate to the consensus DNA binding site TGAg/cTCA in the promoter region of target genes (7). The main structural difference between the various Fos family members is the presence of a transactivation motif at the C-terminal parts of c-Fos and Fos B, which is absent in Fra-1 and Fra-2. These motifs are directly involved in transcriptional activation and critical for the transformation capacity of Fos proteins.

The combination of these dimers varies with the expression and activation level of the individual components according to cell type, environmental factors and phases of the cell cycle $(8,9)$. Thus, Fos and Jun proteins are involved in a variety of dynamically changing protein-protein interactions binding on and off the DNA.

Several experimental systems have demonstrated that increased cell proliferation, malignant transformation and enhanced invasiveness are accompanied by changes in AP-1 complex composition (10-12). It was shown, that Fra-1 and pc-Jun contribute to the development of an aggressive phenotype in breast cancer cells (13-15). 
Besides the apparently important role in breast cancer cell growth and transformation, little is known regarding the function of AP-1 in ovarian cancer.

The aim of the current study was to characterize the role of the different AP-1 proteins in ovarian cancer. Therefore, we analyzed the expression of Jun and Fos proteins in benign ovarian tumors, ovarian tumors with low malignant potential (borderline tumors) and invasive ovarian tumors with their respective metastases. In addition, five human ovarian cancer cell lines with different invasive potential were investigated for cell proliferation, motility and invasion with respect to the expression of Jun and Fos proteins.

\section{Materials and methods}

Tissue samples and patient characteristics. A total of 56 ovarian tumor samples were included in the study: 31 invasive ovarian carcinomas (23 serous, 2 mucinous, 2 endometrioid tumors and 4 samples of mixed differentiation), 13 metastases [10 serous, 2 mucinous, 1 mixed differentiation from the omentum $(n=10)$, ileum $(n=1)$, peritoneum $(n=1)$ and pancreas $(n=1)], 4$ ovarian tumors of low malignant potential (borderline tumors, 2 serous, 2 mucinous) and 8 benign ovarian tumors (4 mucinous cystadenomas, 4 Brenner tumors).

Fresh-frozen samples were obtained intraoperatively and immediately stored at $-80^{\circ} \mathrm{C}$ at the University Medical Center Hamburg-Eppendorf between 1993 and 2002. All patients gave written informed consent to access their tissue and review their medical records according to our Investigational Review Board and Ethics Committee guidelines.

Of the primary invasive tumors, 5 cases were diagnosed in stage I, 2 in stage II, 12 in stage III and 10 in stage IV. Most of the tumors were poorly differentiated $(20 \mathrm{G} 3,8 \mathrm{G} 2$ and $3 \mathrm{G} 1$. The median age of patients with invasive ovarian cancer was 65.0 years (range $27-83$ years), patients with benign tumors had a median age of 50.5 years (range $30-70$ years).

Cell lines. The ovarian cancer cell lines OC 2, Ovcar 3, 4, 5 and 8, as well as the two breast cancer cell lines MDA-MB231 and MCF-7 serving as controls, were cultivated under standard cell culture conditions, as previously described (16).

Cell growth assay. Cellular proliferation was determined using the cell proliferation kit I (MTT, Roche Diagnostics, Mannheim, Germany) according to the manufacturer's instruction. The initial cell density was 2,500 cells per well for each cell line with three replicates per time point. Proliferation of each cell line was measured at 24, 48 and $72 \mathrm{~h}$. Each experiment was repeated three times. The extinction values at $540 \mathrm{~nm}$ obtained after $24 \mathrm{~h}$ were set as $100 \%$ and the relative values at later time points were calculated in relation to this value.

Wound healing assay. A modified wound healing assay was performed according to Misra et al (17). All cell lines were cultured in growth medium with $10 \%$ fetal calf serum (FCS) in 6-well plates to $>90 \%$ confluence. Cells were rinsed with serum-free medium and further incubated in growth medium (5\% FCS). 'Injury lines' $(\sim 0.5 \mathrm{~mm})$ were scratched with sterile pipette tips. The wound healing effect at the cell-free wound area was documented by microscopy (Zeiss, Jena, Germany) and a digital camera (Leica, Solms, Germany) at different time points after scratching $(4,8,11,24,30,39$ and $48 \mathrm{~h}$ ) and the time to gap closure was determined.

Invasion assay. The invasive potential of each cell line was tested using BD Biocoat Matrigel ${ }^{\mathrm{TM}}$ Invasion chambers (24-well plates, BD Bioscience, Heidelberg, Germany) with 50,000 cells per well according to the manufacturer's instruction, but without using a chemo-attractant in order to analyze invasion instead of chemo-attraction. Highly invasive MDA-MB231 cells were used as positive control. For comparison, the same number of control chambers without matrigel membrane (BD Bioscience) was used. All tests were performed simultaneously under similar conditions and each experiment was repeated three times. Assays were carried out at $37^{\circ} \mathrm{C}$ in $5 \% \mathrm{CO}_{2}$ for $48 \mathrm{~h}$. The invasive cells, which had passed the membrane, were counted under the microscope in seven identical square fields covering almost the entire membrane area.

Western blot analysis. In order to confirm the histology of the frozen samples, cryo-cut sections were stained with haematoxylin and eosin and the samples were trimmed if necessary in order to obtain tumor cell contents of at least $70 \%$. Protein extraction and Western blot analysis were performed, as described previously (18). Proteins were lysed in ice-cold sample buffer [50 mM Tris $\mathrm{pH} 6.8,1 \%$ sodium dodecyl sulphate (SDS), $10 \%$ sucrose and $10 \mu 1 \mathrm{ml}^{-1}$ protease inhibitor cocktail (Sigma, Taufkirchen, Germany)]. Equal amounts of protein $(20 \mu \mathrm{g})$ of each sample were loaded per well and equal loading was verified by immunoblotting with actin antibodies (Santa Cruz, Heidelberg, Germany). As control samples, proteins from the breast cancer cell line MCF-7 or MDA-MB231 (for Fra-1) were loaded on each gel. After electrophoresis, blotting to polyvinylidene difluoride (PVDF) membranes and overnight incubation at $4^{\circ} \mathrm{C}$ in blocking solution, membranes were incubated for $1 \mathrm{~h}$ at room temperature with the following primary antibodies (all from Santa Cruz): pc-Jun monoclonal antibody KM1 (1:200), Jun B polyclonal antibody 210 (1:100), Jun D polyclonal antibody 329 (1:2000), c-Fos polyclonal antibody 4 (1:1000), Fos B polyclonal antibody 102 (1: 200), Fra-1 polyclonal antibody R-20 (1:400) and Fra-2 polyclonal antibody Q-20 (1:800). In contrast to the other AP-1 proteins, the anti-c-Jun antibody used in our study was specific for the active, phosphorylated protein (pc-Jun). As secondary antibody, peroxidaseconjugated anti-rabbit-IgG (1:4000) was used, which was visualized by chemiluminescence reagent (Super Signal West Pico kit, Pierce, Rockford, IL, USA) using Hyperfilm ECL (Amersham, Braunschweig, Germany). Band intensities were quantified by densitometry (GS-700 Imaging Densito-meter, Bio-Rad, Munich, Germany) and calculated as percent intensity of the control sample after correction for equal actin loading. For Fra-1, where band intensities were relatively low with a high background staining, expression was categorized by visual inspection as negative, low or moderate (Fig. 2).

Statistical analysis. The Chi-square test and Fisher's exact test (two-sided) were used to examine the correlation between the 


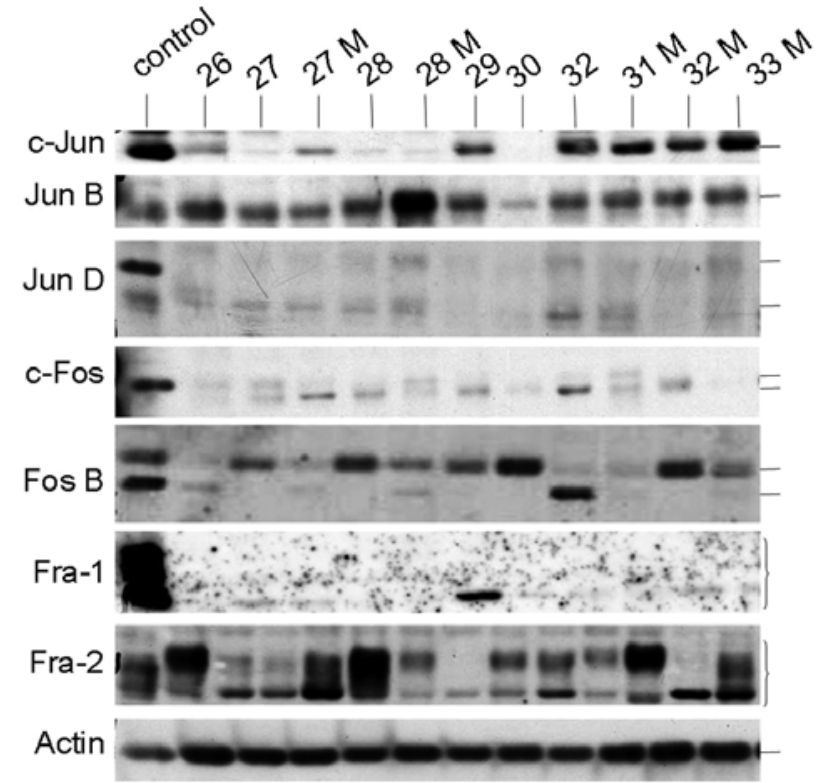

Figure 1. Representative Western blotting result of pc-Jun, Jun B, Jun D, c-Fos Fos B, Fra-1 and Fra-2 expression in 6 primary carcinomas and 5 metastases (indicated by ' $\mathrm{M}$ '). The mammary cell lines MDA-MB231 or MCF-7 (for Fos B) served as control.

analyzed AP-1 proteins and histological or clinical factors. Expression levels of Jun and Fos proteins in the four tumor groups (benign tumors, LMP tumors, primary carcinomas, metastases) were compared by two-tailed t-tests. Probability values $<0.05$ were regarded as statistically significant. All statistical analyses were conducted using SPSS software Version 15 (SPSS Inc., Chicago, IL, USA).

\section{Results}

Expression of AP-1 proteins in ovarian tumors of different invasive potential. The expression of c-Fos, Fos B, Fra-1, Fra-2, pc-Jun, Jun B and Jun D was analyzed by Western blot analysis in 8 benign tumor samples, 4 tumors of low malignant potential (LMP, borderline tumors), 31 primary ovarian carcinomas and 13 metastases. A representative Western blot analysis is shown in Fig. 1.

The results of densitometric evaluation of the expression of c-Fos, Fos B, Fra-2 and the Jun proteins are presented as box plots in Fig. 2A-F. For Fra-1, a semi-quantitative evaluation was performed as shown in Fig. $2 \mathrm{G}$.

In benign tumors, the mean expression levels of pc-Jun, Jun B, Jun D and Fra-2 were significantly lower than those found in invasive carcinomas, whereas for c-Fos, Fos B and Fra-1, similar expression was found. Comparison of the protein expression in invasive carcinomas and tumors of low malignant potential (LMP, borderline tumors) did not yield any significant differences. Tumors from metastatic sites displayed significantly lower c-Fos and Fos B protein levels compared with primary tumors (Fig. 2D and E).

To analyze the association of AP-1 proteins with any clinical (age, FIGO stage) or histological (grading, histological subtype) parameters, the invasive carcinomas were divided into two groups with low and high expression (below and above median) of the respective transcription factor. There were no statistically significant correlations of any of the AP-1 proteins with clinical stage, histological grading, patient's age or histological subtype (not shown).

Expression of AP-1 proteins in ovarian cancer cell lines. To explore the possible molecular basis of differences in biological features of different ovarian carcinoma cell lines, we analyzed the expression levels of c-Fos, Fos B, Fra-1, Fra-2, pc-Jun, Jun B and Jun D by Western blot analysis in five ovarian cancer cell lines. A representative Western blot analysis is shown in Fig. 3C. MDA-MB231 and MCF cells, which are well characterized with respect to AP-1 protein expression, were used as controls. The phosphorylated form of c-Jun (pc-Jun) was weakly expressed in all tested cell lines. Except for Ovcar 3, all cell lines expressed Jun B. Jun D and c-Fos proteins were detectable in all cell lines, whereas Fos B expression was only observed in OC 2 and Ovcar 4, 5 and 8. Fos B expression levels in MCF and Ovcar 5 cells were substantially higher compared to the other cell lines.

MDA-MB231, OC 2 and Ovcar 8 showed extraordinary high expression of Fra-1 protein, whereas in Ovcar 5 and Ovcar 3 cells, only low Fra-1 levels were observed and Fra-1 was not detectable in Ovcar 4 cells. Fra-2 was expressed at different levels in all cell lines with the lowest expression in Ovcar 3 cells.

Invasion and motility of ovarian cancer cell lines. The invasive capacity and motility was tested in matrigel invasion assays, serving as an artificial basal membrane. As expected, MDA-MB231 cells were highly invasive (Fig. 3A). Compared to this cell line, the invasive potential of the ovarian carcinoma cell lines OC 2, Ovcar 3, 5 and 8 was low and Ovcar 4 cells were not invasive (zero cells per counting area).

In contrast to the control cells, the number of OC 2 cells passing through the matrigel membrane exceeded the number of cells counted with control inserts (with a porous bottom, but without a matrigel layer) with mean values of 15 cells (matrigel) and 6 cells (control) per counting area. This apparently paradoxical effect points to stimulation of cell migration and invasion by ingredients of the matrigel. The same was observed for Ovcar 8 cells $(5$ cells per counting area on matrigel and only 2 cells on control inserts). With Ovcar 3 and 5, lower cell numbers were counted on matrigelcoated inserts compared to control inserts. These observations are also reflected by differences in relative invasion (number of invasive cells divided by number of cells passing the control inserts $x$ 100) which was highest in OC $2(250 \%)$ and Ovcar 8 cells $(250 \%)$ compared to Ovcar $5(55 \%)$, Ovcar $3(40 \%)$ and Ovcar $4(0 \%)$.

The cell motility was analyzed in a classical wound healing proliferation assay. The times to gap closure are shown in Fig. 3B. Gap-closure for Ovcar 5 cells was relatively fast $(11 \mathrm{~h})$ compared to the other cell lines. Gap closure for Ovcar 3, Ovcar 4 and Ovcar 8 was completed after $30 \mathrm{~h}$. The migration rate for OC 2 cells was the slowest with gap closure after $48 \mathrm{~h}$.

Proliferation of ovarian cancer cell lines. In MTT proliferation assays, Ovcar 3 cells showed the highest rate of growth. The mean increase in cell numbers was $184 \%$ after $48 \mathrm{~h}$ and 

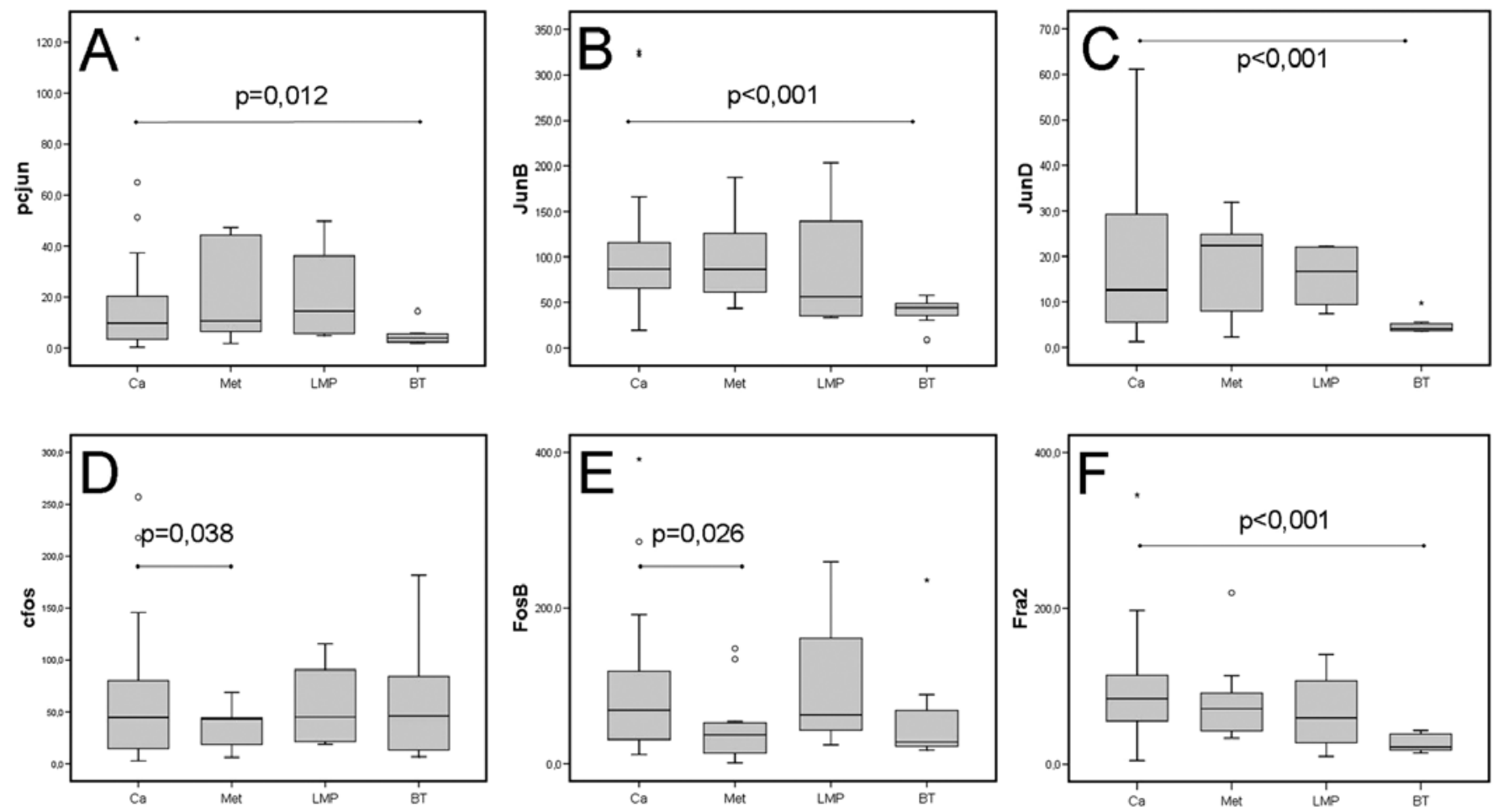

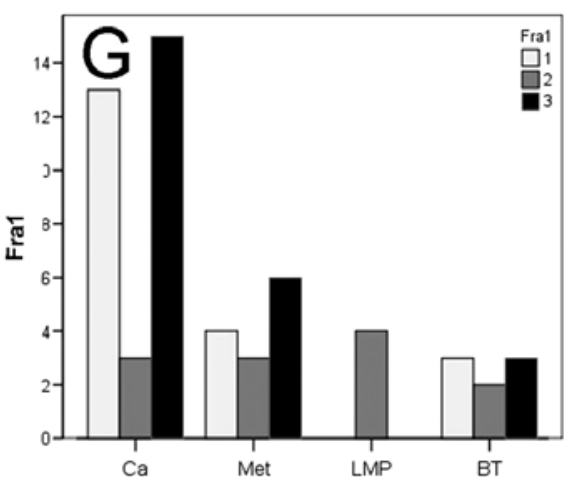

$302 \%$ after $72 \mathrm{~h}$. The growth rate of Ovcar 5 and 8 cells was 178 and $135 \%$ after $24 \mathrm{~h}$ and 245 and $234 \%$ after $72 \mathrm{~h}$, respectively. The proliferation rates of OC 2 and Ovcar 4 cells were as follows: 138 and $141 \%$ after $48 \mathrm{~h}$ and 205 and 204\% after $72 \mathrm{~h}$ (Fig. 3D).

\section{Discussion}

To characterize the role of AP-1 proteins in ovarian cancer, we analyzed the expression of Jun and Fos proteins in benign ovarian tumors, tumors with low malignant potential and invasive ovarian tumors with their respective metastases. In addition, five human ovarian cancer cell lines with different invasive potential were examined for cell proliferation, motility and invasion with respect to the expression of Jun and Fos proteins, representing the first comprehensive investigation of all Jun and Fos proteins in ovarian cancer. We were able to show that AP-1 proteins are differentially expressed in ovarian tumors of various malignant potential and their metastases as well as in different ovarian cancer cell lines, suggesting a possible role of AP-1 proteins in ovarian cancer progression.
Figure 2. Comparison of pc-Jun, Jun B, Jun D, c-Fos, Fos B, Fra-1 and Fra-2 expression in ovarian primary carcinomas $(\mathrm{Ca})$, metastases (Met), borderline tumors (LMP) and benign tumors (BT). Box plots representing relative expression values calculated after densitometry are shown (A-F) except for Fra-1 (G), where a semi-quantitative evaluation was performed and the Fra-1 expression was classified as negative (1), low (2) and moderate (3). Differences were calculated by t-test.

Many in vitro and in vivo experiments as well as analyses of tumor tissues have shown that members of the Jun family (c-Jun, Jun B and Jun D) and Fos family (c-Fos, Fos B, Fra-1 and Fra-2) are able to regulate tumor growth and progression $(14,19,20)$. Due to their ability to form a large number of homo- and heterodimers with different DNA-binding potential and promoter specificity, changes in the expression levels of individual AP-1 family members can lead to variations in the regulation of target genes $(21,22)$.

In breast cancer cell lines, different expression levels of AP-1 proteins, especially Fos B and Fra-1, reflect different biological properties: Fra-1 is overexpressed in highly invasive, estrogen receptor (ER)-negative cell lines with mesenchymal morphology, whereas Fos B is detected in ERpositive, epithelial, less invasive cells $(13,18)$. In transfection and silencing experiments, Fra-1 and Fra-2 overexpression enhanced the invasive potential of breast cancer cells $(5,9,22)$. Moreover, in tumor tissue samples, poorly differentiated carcinomas were characterized by loss of Fos B expression, but relatively high Fra-1 and Fra-2 protein levels $(12,18)$. Increased Fra-1 expression has also been implicated in the etiology of colorectal, lung, thyroid, esophageal and pancreatic 
A
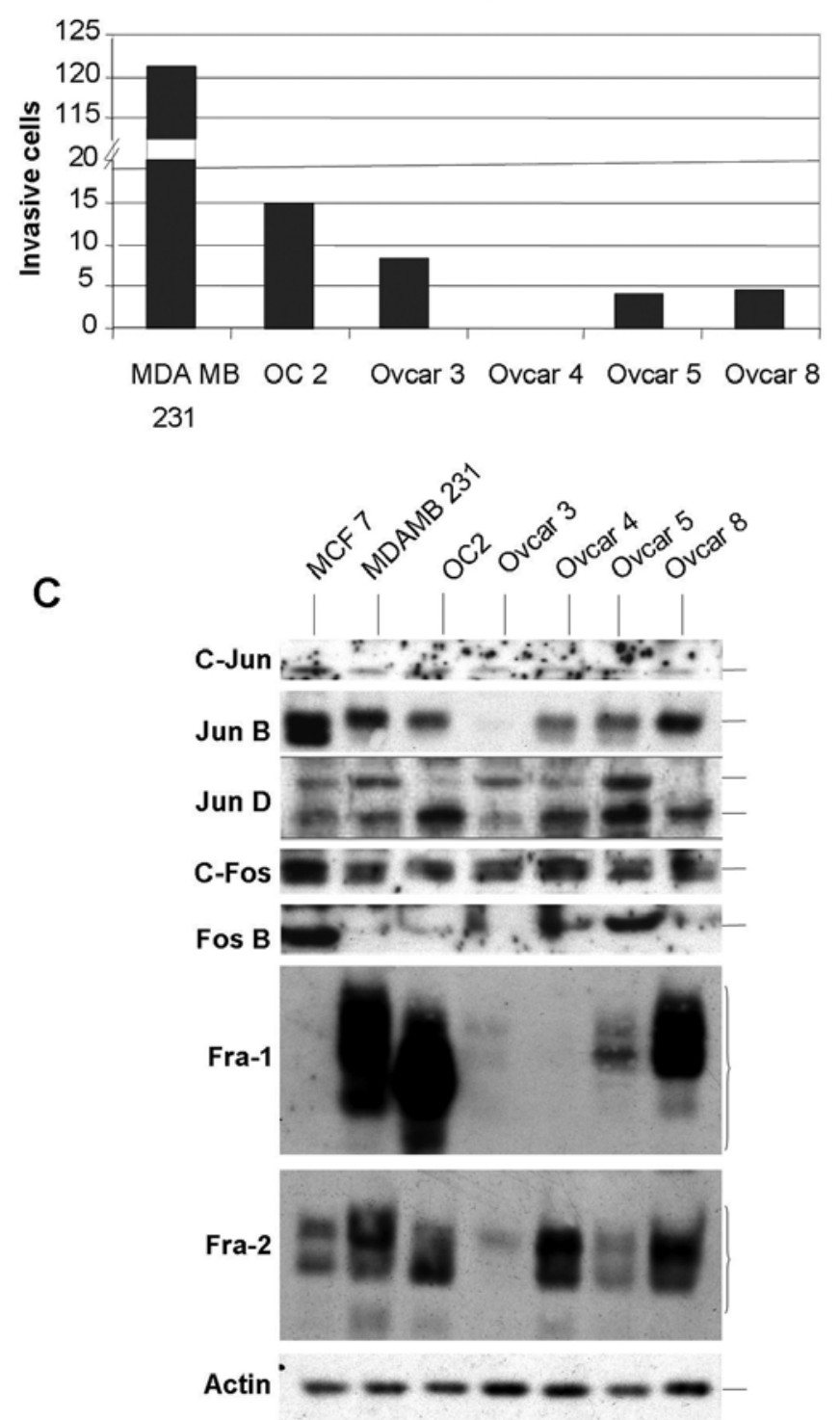

B

Cell Proliferation Wound Healing Assay

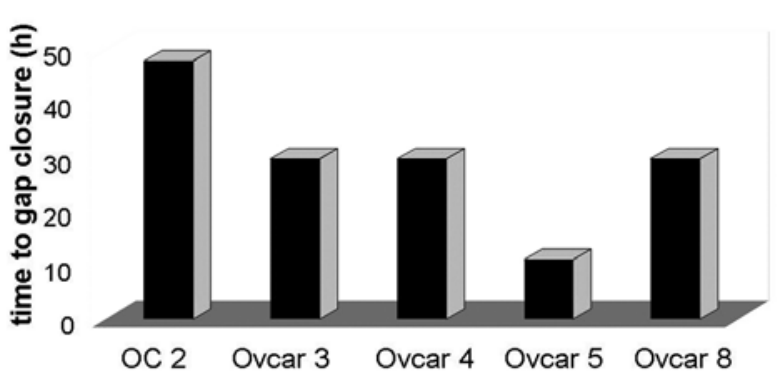

D

Cell Proliferation MTT Assay

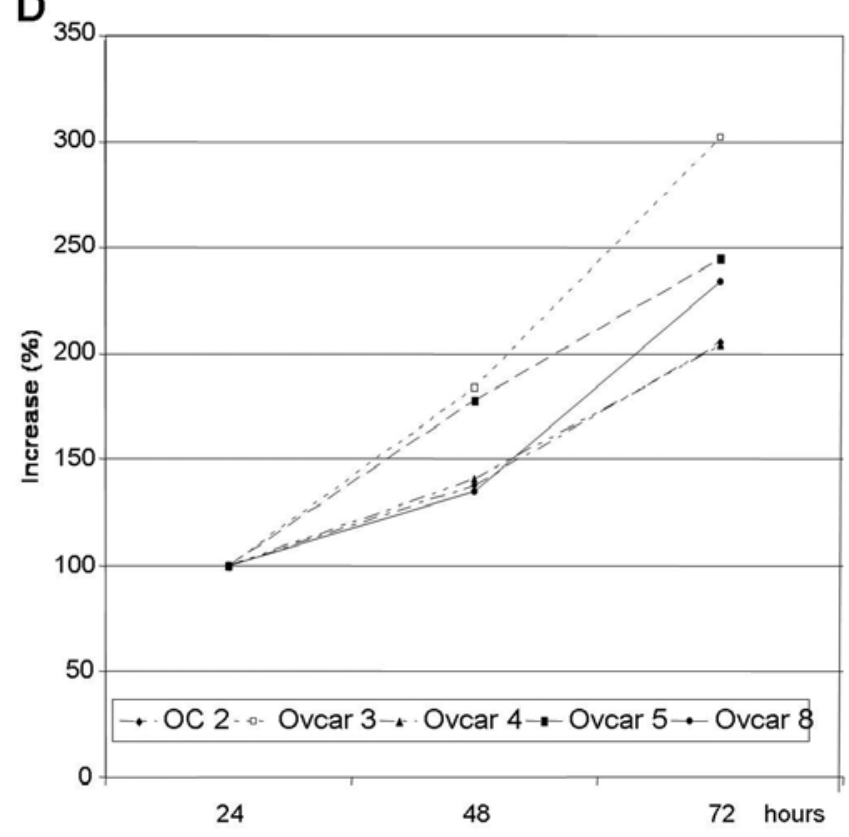

Figure 3. Comparison of different ovarian cancer cell lines. The breast cancer cell lines MDA-MB231 and MCF-7 were used as controls. (A) Invasive potential analyzed by Matrigel invasion assay. Invasive cells were counted in seven identical square fields. Mean values of three independent experiments are shown. (B) Analysis of cell migration by wound healing assays. The time to gap closure is displayed. (C) Representative Western blot analysis of all Fos and Jun proteins in five different ovarian cancer cell lines. (D) MTT proliferation assay. The extinction values obtained after $24 \mathrm{~h}$ were set as $100 \%$ for each cell line and the relative proliferation at each time point was calculated. Mean values of three independent proliferation assays are shown.

carcinoma cells (reviewed in ref. 14). In contrast to the increasing knowledge on the importance of AP-1 proteins in breast cancer, information regarding their potential role in ovarian cancer is limited and inconclusive.

Experimental results on rat ovarian surface epithelial (ROSE) cells suggest that Fra-1 might be involved in ovarian tumorigenesis: after transformation by mutant K-Ras, Fra-1 was up-regulated $>100$-fold (23). $\alpha(\mathrm{v}) B 3$ integrin expression in ovarian OV-MZ-6 cells increases Fra-1 expression and promotes adhesion to vitronectin as well as cell motility (24). In contrast to these results, the analysis of tumor tissue points to a potential role of c-Fos down-regulation in ovarian cancer progression: Gene expression profiling of borderline (LMP-) tumors and carcinomas of different histological grades revealed that borderline and G1 tumors are characterized by a relatively high and uniform c-Fos expression compared to the moderately
(G2) or poorly (G3) differentiated carcinomas (25). In a similar investigation on serous tumors, Gilks et al found a $>5$-fold higher c-Fos expression in LMP tumors compared to invasive ovarian cancer (26). In a series of 101 patients with epithelial ovarian cancer, we recently demonstrated an unfavourable outcome, if intratumoral c-Fos expression was low (27).

The current study represents the first comprehensive investigation of all Jun and Fos proteins in ovarian tumors of different malignant potential and ovarian carcinoma cell lines. Protein expression of the Jun family members was significantly lower in benign tumors than in LMP-tumors as well as invasive carcinomas and their metastases. However, overexpression of these transcription factors in carcinomas was not associated with other clinical or histological factors.

In contrast to Jun proteins, c-Fos expression was downregulated in ovarian cancer and there was a significantly lower 
expression in metastatic lesions compared to primary tumors. This observation corresponds well with previously published RNA expression profiling and intratumoral protein expression, indicating that ovarian tumor progression is accompanied by down-regulation of c-Fos. However, in the current study we could not find a statistically significant difference between LMP lesions and invasive tumors, which might be explained by the relatively low number of borderline tumors in our experiments. Similar to c-Fos, Fos B expression in metastases was significantly lower than in primary ovarian carcinomas, but both factors were not associated with other clinicopathologic parameters. In contrast to breast cancer, we did not find an association of Fos B expression with a welldifferentiated phenotype. However, the low expression level in metastatic lesions suggests that tumor cell progression might also be accompanied by Fos B down-regulation in ovarian cancer. Fra-2 expression was similar to Jun proteins, with low levels in benign tumors, a significantly higher expression in carcinomas and no correlation with clinicopathologic markers in this group.

Fra-1 expression was undetectable or low in ovarian carcinomas and positive results were associated with poor differentiation, which is in line with our previously published results in breast cancer (18). However, this correlation was not statistically significant in ovarian cancer. A common feature in both tumor types is the extremely strong Fra-1 expression in some cell lines (MDA-MB231 in breast cancer, OC 2 and Ovcar 8 in ovarian cancer) which to this extent is never seen in tumor tissue obtained from patients. In comparison to MDA-MB231 cells, these two ovarian cancer cell lines were less invasive and motile, but the ratio of invasive to motile cells was higher than in the other ovarian cancer cell lines. Other than the above, we could not find a correlation between the expression level of any of the AP-1 proteins and the proliferative, migratory or invasive potential of the respective ovarian cell lines.

Our results suggest differential roles of the AP-1 family members, particularly the Fos proteins, in breast and ovarian cancer. In contrast to previous observations in breast cancer, we could not demonstrate an important role of Fra- 1 in the regulation of ovarian tumor invasion and progression. This could partially be explained by the fact that, compared to mammary carcinomas, ovarian cancer progression is not primarily characterized by invasion into surrounding tissue. Instead, detached tumor cells spread extensively within the peritoneal cavity, leading to peritoneal carcinomatosis that does not invade the underlying tissues.

In summary, this study documents for the first time that AP-1 proteins are differentially expressed in benign ovarian tumors, tumors with low malignant potential and epithelial ovarian carcinomas and metastases. In combination with the previously shown prognostic significance of c-Fos expression in ovarian cancer, these results suggest a potential role of AP-1 proteins as clinically useful markers. Thus, further evaluation within the setting of prospective clinical trials is highly desirable.

\section{Acknowledgements}

We thank Gabriele Rieck, Sylke Krenkel, Katrin Beck and Susanne Feldhaus for excellent technical assistance.

\section{References}

1. American-Cancer-Society Cancer Facts and Figures, 2007.

2. du Bois A, Luck HJ, Meier W, Adams HP, Mobus V, Costa S, Bauknecht T, Richter B, Warm M, Schroder W, Olbricht S, Nitz U, Jackisch C, Emons G, Wagner U, Kuhn W and Pfisterer J: A randomized clinical trial of cisplatin/paclitaxel versus carboplatin/paclitaxel as first-line treatment of ovarian cancer. J Natl Cancer Inst 95: 1320-1329, 2003.

3. Deanne NG, Parker MA and Beauchamp RD: Cell proliferation: a matter of time and place. Surgery 138: 1-7, 2005.

4. Yamaguchi $\mathrm{H}$, Wyckhoff $\mathrm{J}$ and Condeelis $\mathrm{J}$ : Cell migration in tumors. Curr Opin Cell Biol 17: 559-564, 2005.

5. Kustikova O, Kramerov D, Grigorian M, Berezin V, Bock E, Lukanidin E and Tulchinsky E: Fra-1 induces morphological transformation and increases in vitro invasiveness and motility of epithelioid adenocarcinoma cells. Mol Cell Biol 18: 7095-7105, 1998.

6. Ozanne BW, Spence HJ, McGarry LC and Hennigan RF: Transcription factors control invasion: AP-1 the first among equals. Oncogene 26: 1-10, 2007.

7. Curran T and Franza BJ: Fos and Jun: the AP-1 connection. Cancer Res 55: 395-397, 1988.

8. Chinenov Y and Kerppola TK: Close encounters of man kinds: Fos-Jun interactions that mediate transcription regulatory specifity. Oncogene 20: 2438-2452, 2001.

9. Belguise K, Kersual N, Galtier F and Chalbos D: FRA-1 expression level regulates proliferation and invasivness of breast cancer cells. Oncogene 24: 1434-1444, 2005.

10. Smith LM, Wise SC, Hendricks DT, Sabichi AL, Bos T, Reddy P, Brown PH and Birrer MJ: cJun overexpression in MCF-7 breast cancer cells produces tumorigenic, invasive and hormone resistant phenotype. Oncogene 18: 6063-6070, 1999.

11. Soto U, Denk C, Finzer P, Hutter K-J, Hausen H and Rösl F: Genetic complementation to non-tumorigenecity in cervicalcarcinoma cells correlates with alterations in AP-1 composition. Int J Cancer 86: 811-817, 2000.

12. Milde-Langosch K, Röder H, Andritzky B, Aslan B, Hemminger G, Brinkmann G, Bamberger CM, Löning T and Bamberger AM: The role of the AP-1 transcription factors c-Fos, FosB, Fra-1 and Fra-2 in the invasion process of mammary carcinomas. Breast Cancer Res Treat 86: 139-152, 2004.

13. Zajchowski D, Bartholdi M, Gong Y, Webster L, Liu HL, Munishkin A, Beauheim C, Harvey S, Ethier SP and Johnson PH: Identification of gene expression profiles that predict the aggressive behavior of breast cancer cells. Cancer Res 61: 5168-5178, 2001

14. Milde-Langosch K: The Fos family of transcription factors and their role in tumorigenesis. Eur J Cancer 41: 2449-2461, 2005.

15. Tkach V, Tulchinsky E, Lukanidin E, Vinson C, Bock E and Berezin V: Role of the Fos family members, c-Fos, Fra- 1 and Fra-2, in regulation of cell motility. Oncogene 22: 5045-5054, 2003.

16. Milde-Langosch K, Bamberger A-M, Goemann C, Rössing E, Rieck G, Kelp B and Löning T: Expression of cell-cycle regulatory proteins in endometrial carcinomas: correlations with hormone receptor status and clinicopathologic parameters. J Cancer Res Clin Oncol 127: 537-544, 2001.

17. Misra A, Lim RPZ, Wu Z and Thanabalu T: N-WASP plays a critical role in fibroblast adhesion and spreading. Biochem Biophys Res Commun 364: 908-912, 2007.

18. Bamberger A-M, Methner C, Lisboa BW, Städtler C, Schulte HM, Löning $\mathrm{T}$ and Milde-Langosch K: Expression pattern of the AP-1 familiy in breast cancer: association of FosB expression with a well-differentiated, receptor-positiv tumor phenotype. Int J Cancer 84: 533-538, 1999.

19. Jochum W, Passegué E and Wagner EF: AP-1 in mouse development and tumorigenesis. Oncogene 20: 2401-2412, 2001.

20. Ozanne BW, McGarry LC, Spence HJ, Johnston I, Winnie J, Meagher L and Stapleton G: Transcriptional regulation of cell invasion: AP-1 regulation of a multigenic invasion programme. Eur J Cancer 36: 1640-1648, 2000.

21. Ryseck R-P and Bravo R: c-Jun, JunB, and JunD differ in their binding affinities to AP- 1 and CRE consensus sequences: effect of fos proteins. Oncogene 6: 533-542, 1991.

22. Milde-Langosch K, Janke S, Wagner I, Schröder C, Streichert T, Bamberger A-M, Jänicke $F$ and Löning T: Role of Fra- 2 in breast cancer: influence on tumor cell invasion and motility. Breast Cancer Res Treat 107: 337-347, 2008. 
23. Tchernitsa OI, Sers C, Zuber J, Hinzmann B, Grips M, Schramme A, Lund P, Schwendel A, Rosenthal A and Schäfer R: Transcriptional basis of KRAS oncogene-mediated cellular transformation in ovarian epithelial cells. Oncogene 23: 4536-4555, 2004.

24. Hapke S, Kessler H, Luber B, Benge A, Hutzler P, Hofler H Schmitt $M$ and Reuning U: Ovarian cancer cell proliferation and motility is induced by engagement of integrin alpha(v)beta3/ vitronectin interaction. Biol Chem 384: 1073-1083, 2003.

25. Meinhold-Heerlein I, Bauerschlag D, Hilpert F, Dimitrov P, Sapinoso LM, Orlowska-Volk M, Bauknecht T, Park TW, Jonat W, Jacobsen A, Sehouli J, Luttgers J, Krajewski M, Krajewski S, Reed JC, Arnold N and Hampton GM: Molecular and prognostic distinction between serous carcinomas of varying and malignant potential. Oncogene 24: 1053-1065, 2004.
26. Gilks CB, Vanderhyden BC, Zhu S, van Rijn M and de Longarcre TA: Distinction between serous tumors of low malignant potential and serous carcinomas based on global mRNA expression profiling. Gynecol Oncol 96: 684-694, 2005.

27. Mahner S, Baasch C, Schwarz J, Hein S, Wölber L, Jänicke F and Milde-Langosch $\mathrm{K}$ : C-Fos expression is a molecular predictor of progression and survival in epithelial ovarian carcinoma. Br J Cancer 99: 1269-1275, 2008. 